\title{
CLASSIFICATION OF DIFFERENTIALS ON QUANTUM DOUBLES AND FINITE NONCOMMUTATIVE GEOMETRY
}

\author{
S. MAJID
}

\begin{abstract}
We discuss the construction of finite noncommutative geometries on Hopf algebras and finite groups in the 'quantum groups approach'. We apply the author's previous classification theorem, implying that calculi in the factorisable case correspond to blocks in the dual, to classify differential calculi on the quantum codouble $\mathbb{C}(G) \prec \mathbb{C} G=D^{*}(G)$ of a finite group $G$. We give $D^{*}\left(S_{3}\right)$ as an example. We explain the geometric meaning of the Woronowicz construction for higher forms in terms of a Hodge ${ }^{*}$ operator.
\end{abstract}

\section{INTRODUCTION}

Coming out of quantum groups has emerged a 'quantum groups approach to noncommutative geometry' somewhat different from the operator algebras one but with some points of contact with that. This paper is an introduction to the quantum groups approach, which is based on building up the different layers of geometry: the differential structure, line bundles, frame bundles, etc. eventually arriving at spinors and a Dirac operator as naturally constructed and not axiomatically imposed as a definition of the geometry (as in Connes spectral triple approach). The quantum groups methods are very algebraically computable and hence should be interesting even to readers coming from the spectral triple side. We demonstrate this on the nice example of the Drinfeld quantum double of a finite group, which is in fact a cross coproduct $\mathbb{C}(G)<\mathbb{C} G$.

In fact, the convergence and interaction between the two approaches is a very important recent development and this paper, as my lecture, aims to bridge between them and hence between the two weeks of the conference at the Banach Center. This is the topic of Section 2. We describe the 'dictionary' as well as the differences between the two approaches and discuss issues that appear to have led to confusion in the literature.

We will then describe the construction of bicovariant differentials in detail as the first step in the quantum groups approach. We start in Section 3 with a review of the seminal work of Woronowicz [1]. Section 4 is my own classification 2 for factorisable quantum groups such as $\mathbb{C}_{q}[G]$. Let us mention that in addition there are the BeggsMajid classification theorem [3] for bicrossproduct quantum groups $\mathbb{C}(M) \backsim \mathbb{C} G$ and the Majid-Oeckl twisting theorem[16] (which covers most triangular quantum groups), which altogether pretty much cover all classes of interest. In this way the first layer of geometry, choosing the differential structure, is more or less well understood in the quantum groups approach.

Date: April, 2002.

1991 Mathematics Subject Classification. 58B32, 58B34, 20 C05.

The author is a Royal Society University Research Fellow. 
Section 5 contains our new result as a worked application: differential calculi on $D^{*}(H)$ where $H$ is a finite-dimensional Hopf algebra and $D^{*}(H)$ is the dual of its Drinfeld double[5]. Even the case $H=\mathbb{C} G$, the group algebra on a finite group, is interesting as we demonstrate for $G=S_{3}$.

Section 6 returns to the Woronowicz theory, now for the higher differential forms, i.e. the entire DGA (differential graded algebra). We present the 'braided factorial' formulation of the Woronowicz antisymmetrizers due to the author[6] and BespalovDrabant[7]. It allows us to explain (as far as I know for the first time) the 'true meaning' of the Woronowicz construction as the minimal relations needed for an invertible Hodge * operator, a point of view which is perhaps the second result of the present paper.

\section{Comparing the operator THEORY AND QUANTUM GROUPS APPROACHES}

A dictionary comparing the two approaches appears in Table 1, and let me say right away that we are not comparing like with like. The Connes approach is mathematically purer and has deeper theorems, while the quantum groups one is more organic and experience-led. Both approaches of course start with an algebra $M$, say with unit (to keep things simple), thought of as 'functions' but not necessarily commutative. The algebra plays the role of a topological space in view of the Gelfand-Naimark theorem. I suppose this idea goes back many decades to the birth of quantum mechanics. Integration of course is some kind of linear functional. In either approach it has to be made precise using analysis (and we do not discuss this here) but at a conceptual level there is also the question: which functional? The cyclic cohomology approach gives good motivation to take here an element of $H C^{0}(M)$, i.e. a trace functional as an axiom. We don't have a good axiom in the quantum groups approach except when $M$ is itself a quantum group, when translation invariance implies a unique one. In examples, it isn't usually a trace. For other spaces we would hope that the geometry of the situation, such as a (quantum group) symmetry would give the natural choice. So here we already see a difference in scope and style of the approaches. Also, since we usually proceed algebraically in the quantum groups approach, we work over $k$ a general field.

Next, also common, is the notion of 'differential structure' specified as an exterior algebra of differential forms $\Omega$ over $M$. An example is the universal differential calculus $\Omega_{\text {univ }}$ going back to algebraic topology, Hochschild cohomology etc. in the works of Quillen, Loday, Connes, Karoubi and others. Here

$$
\Omega_{\text {univ }}^{1}=\operatorname{ker}(\cdot), \quad \mathrm{d} f=f \otimes 1-1 \otimes f, \quad \forall f \in M,
$$

where $\cdot: M \otimes M \rightarrow M$ is the product. Similarly for higher degrees. All other exterior algebras are quotients, so to classify the abstract possibilities for $\Omega$ as a DGA we have to construct suitable differential graded ideals. This is the line taken in [8] and an idea explained there is to start with a spectral triple $(\rho(M), \mathcal{H}, D)$ as a representation of $\Omega_{\text {univ }}$, where d is represented by commutator with an operator $D$ called 'Dirac', and then to divide by the kernel. This does not however, usually, impose enough constraints for a reasonable exterior algebra $\Omega(M)$, e.g. it is often not finite-dimensional over $M$, so one forces higher degrees to be zero, as well as additional relations with the aid of a cyclic cocycle of the desired top degree $d$ corresponding to a 'cycle' $\int: \Omega \rightarrow \mathbb{C}$. For an orientation there is also a $\mathbb{Z}_{2}$ grading operator (trivial in the odd case) and a charge conjugation operator, and Connes requires a certain Hochschild cycle of degree $d$ whose image in the spectral 


\begin{tabular}{|c|c|c|}
\hline Classical & Operator approach & Quantum groups approach \\
\hline $\begin{array}{c}\text { topol. space } \\
\text { integration } \\
\text { differential calc. } \\
\text { contruction of : } \\
\text { inner calculus } \\
\text { top form } \\
\end{array}$ & $\begin{array}{c}\text { algebra } M \\
\int: M \rightarrow \mathbb{C}, \text { trace } \\
\text { DGA } \Omega, \mathrm{d} \\
\text { spe. triple }(\rho(M), \mathcal{H}, D) \\
\quad- \\
\text { impose cycle }\end{array}$ & $\begin{array}{c}\text { algebra } M \\
\int: M \rightarrow k, \quad \text { symmetry } \\
\text { bimodule } \Omega^{1} \Rightarrow \text { DGA } \Omega \\
\text { classify all by symmetry } \\
\text { typ. } \exists \theta: \mathrm{d}=[\theta,\} \\
\text { typ. } \exists \text { Top }\end{array}$ \\
\hline $\begin{array}{l}\text { principal bundle } \\
\text { vector bundle } \\
\text { connection } \\
\text { Chern classes }\end{array}$ & $\begin{array}{c}- \\
\text { projective module }(\mathcal{E}, e) \\
\nabla \\
\text { Chern - Connes pairing }\end{array}$ & $\begin{array}{c}\left(P, \Omega^{1}(P), A, \Omega^{1}(A), \Delta_{R}\right) \\
H \text { quantum group fiber } \\
\mathcal{E}=(P \otimes V)^{A}, \text { typ. } \exists e \\
\Omega^{1}(P)=\Omega_{\text {hor }}^{1} \oplus \Omega_{\text {ver }}^{1} \\
\Leftrightarrow \omega: \Lambda^{1}(A) \rightarrow \Omega^{1}(P) \Rightarrow D_{\omega} \\
-\end{array}$ \\
\hline $\begin{array}{l}\text { frame bundle } \\
\text { metric } \\
\text { Levi - Civta } \\
\text { Ricci tensor } \\
\text { spin bundle } \\
\text { Dirac operator } \\
\text { Hodge star }\end{array}$ & $\begin{array}{c}- \\
\|[D, M]\| \Rightarrow d(\psi, \phi) \\
\text { contained in } D \\
\text { assumed }(D, \mathcal{H}) \\
\text { in }\|\|+\text { orientation }\end{array}$ & $\begin{array}{c}\text { princ. } P+\text { soldering } \Rightarrow \mathcal{E} \cong \Omega^{1}(M) \\
g \in \Omega^{1} \otimes_{M} \Omega^{1} ; \text { coframing } \\
D_{\omega}+\text { soldering } \Rightarrow \nabla_{\omega} \\
\mathrm{d} \omega+\omega \wedge \omega \Rightarrow \text { Ricci } \\
\text { assoc. to frame bundle } \\
\left(\mathrm{d}, \gamma_{a}, \omega\right) \Rightarrow \not D \\
g, \text { Top } \Rightarrow *\end{array}$ \\
\hline
\end{tabular}

TABLE 1. Two approaches to noncommutative geometry

triple representation is the grading operator. This data together with the operator norm and a K-theoretic condition for Poincaré duality subsumes the role played in geometry by the usual Hodge ${ }^{*}$ operator and the volume form.

Here again the quantum groups approach is less ambitious and we don't have a general construction. Instead we classify all possible $\Omega^{1}(M)$, typically restricted by some symmetry to make the problem manageable. We postpone $\Omega^{2}(M)$ and higher till later. Thus a first order calculus over $M$ is a nice notion all by itself:

- An $M-M$-bimodule $\Omega^{1}$

- A linear map d : $M \rightarrow \Omega^{1}$ such that $\mathrm{d}(f g)=(\mathrm{d} f) g+f \mathrm{~d} g$ for all $f, g \in M$ and such that the map $M \otimes M \rightarrow \Omega^{1}$ given by $f \mathrm{~d} g$ is surjective.

It means classifying sub-bimodules of $\Omega_{u n i v}^{1}$ to quotient by. In the last 5 years it was achieved on a case-by-case bass for all main classes of quantum groups and a bicovariant calculus (see section 3). Then homogeneous spaces will likewise be constrained by smoothness of desired actions and hence inherit natural choices for their calculi, and so on for an entire noncommutative universe of objects. Then we look at natural choices for $\Omega^{2}(M), \Omega^{3}(M)$ etc. layer by layer and making choices only when needed. There is a key lemma [9] that if $\Omega^{m}(M)$ for $m \leq n$ are specified with $\mathrm{d}$ obeying $\mathrm{d}^{2}=0$ then these have a maximal prolongation to higher degree, where we add in only those relations at higher degree implied by $\mathrm{d}^{2}=0$ and Leibniz. And when $M$ is a quantum group there is a canonical extension to all of $\Omega(M)$ due to [1] and the true meaning of which is Poincaré duality as we shall explain in Section 6. Here the algebraic information in the 'skew symmetrization' used to build the higher exterior algebra typically gives a top form and Hodge $*$ operator. 
Apart from these differences in style the two approaches are again broadly similar at the level of the differential forms and there is much scope for convergence. Let us note one difference in terminology. In the quantum groups approach we call a calculus 'inner' if there is a 1 -form $\theta$ which generates $d$ as graded commutator. This looks a lot like Connes 'Dirac operator' $D$ in the spectral triple representation

$$
\rho_{D}(\mathrm{~d} f)=[D, \rho(f)]
$$

but beware: our $\theta$ is a 1 -form and nothing to do with the geometric Dirac operator $\not D$ that we construct only much later via gamma-matrices, spin connections etc. Moreover $D$ in a spectral triple does not need to be in the image under the representation $\rho_{D}$ of the space of 1 -forms and should not be thought of as a one form.

The next layers of geometry require bundles. In the traditional approach to vector bundles coming from the theorems of Serre and Swann one thinks of these as finitely generated projective modules over $M$. However, in the quantum groups approach, as in differential geometry, we think that important vector bundles should really come as associated to principal ones. Principal bundles should surely have a quantum group fiber so this is the first place where the quantum group approach comes into its own. The Brzezinski-Majid theory of these is based on an algebra $P$ (the 'total space'), a quantum group coordinate algebra $A$, and differential structures on them, a coaction $\Delta_{R}: P \rightarrow P \otimes A$ such that $M=P^{A}$ and an exact sequence[10]

$$
0 \rightarrow P \Omega^{1}(M) P \rightarrow \Omega^{1}(P) \stackrel{\text { ver }}{\longrightarrow} P \otimes \Lambda^{1}(A) \rightarrow 0
$$

which expresses 'local triviality'. Here $\Lambda^{1}(A)$ are the left-invariant differentials in $\Omega^{1}(A)$ and the map ver is the generator of the vertical vector fields. We require its kernel to be exactly the 'horizontal' forms pulled up from the base. It is sometimes thought that this just means a Hopf-Galois extension but actually it is only equivalent to that concept in the geometrically less interesting case of the universal differential calculus. A connection is a splitting of this sequence and characterised by a connection form $\omega$. Of course, we have associated vector bundles $\mathcal{E}=(P \otimes V)^{A}$ for every $H$-comodule $V$, analogous to the usual geometric construction. A connection $\omega$ induces a covariant derivative $D_{\omega}$ with the 'derivation-like' property that one expects for an abstract covariant derivative $\nabla$, so the two approaches are compatible. It is a pleasure to note that this 'quantum groups gauge theory' was the topic our contribution [1] to the Banach Center proceedings from 1995.

Just as the main example in those days of the operator theory approach was the 'noncommutative torus' $T_{\theta}^{2}[12$, with vector bundles classified in [13, the main example for noncommutative bundles in the quantum groups approach was the $q$-monopole bundle [10] over the Podlès 'noncommutative sphere' $S_{q}^{2}$. Probably the first nontrivial convergence between these approaches was in 1997 with the Hajac-Majid projector 14:

$$
e=\left(\begin{array}{cc}
q^{2} b_{3}+1-q^{2} & q b_{+} \\
-b_{-} & 1-b_{3}
\end{array}\right) \in M_{2}\left(S_{q}^{2}\right), \quad e \mathrm{~d} e \cong D_{\omega}, \quad\langle[\tau],[e]\rangle=\tau(\operatorname{Tr}(e))=1
$$

where $\omega$ is the $q$-monopole connection and $\tau$ is the Masuda et al. trace on $S_{q}^{2}$ which had been found in 15 . The pairing is the Chern-Connes one between $H C^{0}$ and 
the class of $e$ as an element of the K-group $K_{0}$. We use the standard coordinates on $S_{q}^{2}$ namely with generators $b_{ \pm}, b_{3}$ and relations

$$
b_{ \pm} b_{3}=q^{ \pm 2} b_{3} b_{ \pm}+\left(1-q^{ \pm 2}\right) b_{ \pm}, \quad q^{2} b_{-} b_{+}=q^{-2} b_{+} b_{-}+\left(q-q^{-1}\right)\left(b_{3}-1\right)
$$

$$
b_{3}^{2}=b_{3}+q b_{-} b_{+} .
$$

In this way we exhibited the lowest charge monopole bundle $\mathcal{E}_{1}$ as a projective module. At the same time, since the Chern-Connes pairing is nonzero, it means that the $q$-monopole bundle is indeed nontrivial as expected. The computations in 14 are algebraic and, moreover, only for the universal differential calculus, but we see that matching up the two approaches is useful even at this level. This projector has sparked quite a bit of interest in recent years. Dabrowski and Landi looked for similar constructions and projectors for $q$-instantons, replacing complex numbers by quaternions. An inspired variant of that became the Connes-Landi projectors for twisted spheres $S_{\theta}^{4}$. In these cases the new idea is (as far as I understand) to work backwards from an ansatz for the form of projector to the forced commutation relations for those matrix entries as required by $e^{2}=e$ and a desired pairing with cyclic cohomology. Note that, as twists, the noncommutative differential geometry of such examples is governed at the algebraic level by the Majid-Oeckl twisting theorem 16$]$.

Finally, we come to the 'top layer' which is Riemannian geometry. In the approach of Connes this is all contained in the 'Dirac operator' $D$ which was assumed at the outset as an axiom. In the quantum groups approach we have been building up the different layers and hope to construct a particular family of $\not D$ reflecting all the choices of differential structure, spin connections etc. that we have made at lower levels. So the two approaches are going in opposite directions. In the table we show the quantum groups formulation of Riemannian geometry introduced by the author in 1997 in [17] and studied recently for finite sets [18], based on the notion of a quantum frame bundle. The main idea is a principal bundle $\left(P, A, \Delta_{R}\right)$ and an $A$-comodule $V$ along with a 'soldering form' $\theta_{V}: V \rightarrow \Omega^{1}(P)$ that ensures that

$$
\Omega^{1}(M) \cong \mathcal{E}=(P \otimes V)^{A}
$$

i.e. that the cotangent bundle really can be identified as an associated bundle to the principal 'frame' one. It turns out that usual notions proceed independently of the choice of $A$, though what $\omega$ are possible and what $\nabla_{\omega}$ they induce depends on the choice of $A$. Here $\nabla_{\omega}: \Omega^{1}(M) \rightarrow \Omega^{1}(M) \otimes_{M} \Omega^{1}(M)$ is $D_{\omega}$ mapped over under the soldering isomorphism. A certain $\bar{D}_{\omega} \wedge \theta_{V}$ corresponds similarly to a torsion tensor. This much is a 'framed manifold' with connection. A framed Riemannian manifold needs in addition a nondegenerate tensor $g \in \Omega^{1}(M) \otimes \Omega^{1}(M)$, which is equivalent to a coframing $\theta_{V^{*}}: V^{*} \rightarrow \Omega^{1}(P)$ via

$$
g=\left\langle\theta_{V} \underset{M}{\otimes} \theta_{V^{*}}\right\rangle,
$$

where we pair by evaluating on the canonical element of $V \otimes V^{*}$, and $\theta_{V^{*}}$ is such that $\mathcal{E}^{*} \cong \Omega^{1}(M)$. Since $\mathcal{E}^{*}$ under the original framing is isomorphic to $\Omega^{-1}(M)$, such a coframing is the same as an isomorphism $\Omega^{-1}(M) \cong \Omega^{1}(M)$. The coframing point of view is nice because it points to a natural self-dual generalization of Riemannian geometry: instead of demanding zero torsion and $\nabla_{\omega} g=0$ as one might usually do for a Levi-Civita connection, one can demand zero torsion and zero cotorsion 
(i.e. torsion with respect to $\theta_{V^{*}}$ as framing). The latter is a skew-version of metric compatibility as explained in 17]. We can also demand symmetry, if we want, in the form

$$
\wedge(g)=0
$$

and we can limit ourselves to $\theta_{V^{*}}$ built from $\theta_{V}$ induced by an $A$-invariant local metric $\eta \in V \otimes V$. Let us also note that if $M$ is parallelizable we can frame with a trivial tensor product bundle and $\theta_{V}, \theta_{V^{*}}$ reduce to a vielbein $e_{V}: V \rightarrow \Omega^{1}(M)$ and covielbein $e_{V^{*}}$. A connection $\omega$ reduces to a 1 -form

$$
\alpha: \Lambda^{1}(A) \rightarrow \Omega^{1}(M),
$$

etc., in keeping with the local picture favoured by physicists.

We are then able to take a different $A$-comodule $W$, say, for spinors. The associated bundle $\mathcal{S}=(P \otimes W)^{A}$ gets its induced covariant derivative from the spin connection $\omega$ on the principal bundle, and in many cases there is a reasonable choice of local metric $\eta$ and of 'gamma-matrices'. We then define the Dirac operator from these objects much as usual. By now the approach is somewhat different from the Connes one and we do not typically obtain something obeying the axioms for $D$. This seems the case even for finite groups, see [18]. The fundamental reason is perhaps buried in the very notion of vector field: in the parallelizable case an $M$-basis $\left\{e_{a}\right\}$ of $\Omega^{1}(M)$ implies 'partial derivatives' $\partial^{a}$ defined by $\mathrm{d} f=\sum_{a} e_{a} \partial^{a}(f)$. These are not usually derivations but more typically 'braided derivations' (e.g. on a quantum group this is shown in [2]). In cases such as the noncommutative torus one has in fact ordinary derivations around. The noncommutative differential calculus is a twist so that the constructions look close to classical. But for $q$-examples and even finite group examples, this is not at all the case. Perhaps this is at the root of the mismatch and may stimulate a way to fix the problem.

\section{Classifying Calculi on general Hopf algebras}

We now focus for the rest of the paper on a small part of the quantum groups approach discussed above, namely just the differential calculus, and for the most part just $\Omega^{1}$. In this section we let $M=A$ be a Hopf algebra over a field $k$. Following Woronowicz[1], a differential structure is bicovariant if:

- $\Omega^{1}$ is a bicomodule with $\Delta_{L}: \Omega^{1} \rightarrow A \otimes \Omega^{1}$ and $\Delta_{R}: \Omega^{1} \rightarrow \Omega^{1} \otimes A$ bimodule maps.

- $\mathrm{d}$ is a bicomodule map.

Here a Hopf algebra means a coproduct $\Delta: A \rightarrow A \otimes A$, a counit $\epsilon: A \rightarrow k$ and an antipode $S: A \rightarrow A$ such that $A$ is a coalgebra, $\Delta$ an algebra map etc. 19. Coalgebras and (bi)comodules are defined in the same way as algebras and (bi)modules but with the directions of structure maps reversed. In the bicovariance condition $A$ is itself a bi(co)module via the (co)product and $\Omega^{1} \otimes A, A \otimes \Omega^{1}$ have the tensor product (bi)module structure. The second condition in particular fully determines $\Delta_{L}, \Delta_{R}$ by compatibility with $\Delta$, so a bicovariant calculus means precisely one where left and right translation expressed by $\Delta$ extend consistently to $\Omega^{1}$. The universal calculus $\Omega_{u n i v}^{1} \subset A \otimes A$ is bicovariant with coactions the tensor product of the regular coactions defined by the coproduct on each copy of $A$.

The result in [1] is that $\Omega^{1}$ in the bicovariant case is fully determined by the subspace $\Lambda^{1}$ of (say) $\Delta_{L}$-invariant 1-forms. Indeed, there is a standard bi(co)module 
isomorphism

$$
A \otimes A \cong A \otimes A, \quad a \otimes b \mapsto a \Delta b
$$

under which $\Omega_{\text {univ }}^{1} \cong A \otimes A^{+}$, where $A^{+}=\operatorname{ker} \epsilon$ is the augmentation ideal or kernel of the counit (classically it would be the functions vanishing at the group identity). The bimodule structure on the right hand side of $(10)$ is left multiplication in the first $A$ from the left and the tensor product of two right multiplications from the right. The bicomodule structure is the left coproduct on the first $A$ from the left and the tensor product of right comultiplication and the right quantum adjoint coaction from the right. Hence we arrive at the classic result:

Proposition 3.1. (Woronowicz) Bicovariant $\Omega^{1}$ are in 1-1 correspondence with quotient objects $\Lambda^{1}$ of $A^{+}$as an $A$-crossed module under right multiplication and the right quantum adjoint coaction.

We recall that an $A$-crossed module means a vector space which is both an $A$-module and a compatible $A$-comodule; the compatibility conditions are due to Radford and correspond in the finite-dimensional case to a module of the Drinfeld double $D(A)=A^{* \text { op }} \bowtie A$ when we view a right coaction of $A$ as a right action of $A^{* \text { op }}$ by evaluation. Given the crossed module $\Lambda^{1}$ we define $\Omega^{1}=A \otimes \Lambda^{1}$ with the regular left(co)modules and the tensor product (co)actions from the right. An introduction for algebraists to this algebraic version of [1] is in [20].

\section{Classification on coquasitriangular Hopf algebras}

After Woronowicz's 1989 work for any Hopf algebra, the next general classification result is my result for factorisable coquasitriangular Hopf algebras such as the coordinate algebras $\mathbb{C}_{q}[G]$ of the Drinfeld-Jimbo quantum groups, presented in Goslar, July 1996 in the plenary session and published in [2]:

Corollary 4.1. (Majid) For the standard $q$-deformations $\mathbb{C}_{q}[G]$ and generic $q$, irreducible $\Omega^{1}$ with a good classical limit are in 1-1 correspondence with nontrivial irreducible representations of $U_{q}(g)$.

The writing in 20] was in a standard physicists style for which the more formal way of writing and certain subtleties (all mentioned in the longer paper) were well-known. It was explained that one should work with coactions rather than actions, etc., and in particular that the classification depends on the ReshetikhinSemenov-Tian-Shanksy theorem that $U_{q}(g)$ is factorisable, which is true over formal powerseries $\mathbb{C}[[\hbar]]$, as well as a Peter-Weyl block decomposition which is true classically and for generic $q$. Hence we only pick up generic calculi that are compatible with formal deformation. On the other hand, this was the first ever classification result of any kind equating calculi to irreducible representations; until then it was known from Jurco 22 that an R-matrix leads to a differential calculus and of course Jurco's construction can be used in any representation of the universal R-matrix $\mathcal{R} \in U_{q}(g) \otimes U_{q}(g)$ to gives a calculus (this was known), but that one could aim at a complete classification like this was totally new at the time. For example, Schmuedgen (who was chairman in my Goslar presentation) and Schueler had at that point classified calculi[23] on $\mathbb{C}_{q}\left[S L_{n}\right]$ of dimension $n^{2}$, which are just variants for one representation. Of these only one has a good $q=1$ limit so there is no contradiction with my result above. The others are in fact just the same but 'twists' 
by root of unity factors and indeed the above corollary is entirely orthogonal to this kind of variation.

The corollary follows from a discussion of a purely algebraic theorem which we explain next. The main ideas are visible even for finite-dimensional Hopf algebras, where all the above subtleties vanish and the results in $[2$ are undeniable:

Theorem 4.2. (Majid) Let $A$ be a finite-dimensional factorisable coquasitriangular Hopf algebra with dual $H$. Bicovariant $\Omega^{1}(A)$ are in 1-1 correspondence with twosided ideals of $\mathrm{H}^{+}$.

Proof. This is [2, Prop. 4.2] which states that the required action of the quantum double on $\Lambda^{1 *} \subset H^{+}$under the quantum Killing form isomorphism $\mathcal{Q}: A^{+} \rightarrow H^{+}$is the left and right coregular representation of two copies of $H$ on $A^{+}$(the coregular action of $H$ on $A$ is obviously adjoint to the regular action of $H$ on $H$ if we wish to phrase it explicitly in terms of $H$, i.e. $\Lambda^{1}$ is isomorphic to a quotient of $H^{+}$by a 2 -sided ideal). $\diamond$

As far as the actual proof in [2] is concerned, there were two ideas. First of all, instead of classifying quotients $\Lambda^{1}$ of $A^{+}$it is convenient to classify their duals or 'quantum tangent spaces' as subcrossed modules $\mathcal{L} \subset H^{+}$. For coirreducible calculi we want irreducible $\mathcal{L}$, but we can also classify indecomposable ones, etc. Of course an $A$-crossed module can be formulated as an $H$-crossed module (the roles of action and coaction are swapped) or $D(H)$ module, so actually we arrive at a self-contained classification for quantum tangent spaces for any Hopf algebra $H$. One has to dualise back (as above) to get back to the left-invariant 1-forms. The second idea was that when $H$ is factorisable the quantum Killing form $\mathcal{Q}=$ $\mathcal{R}_{21} \mathcal{R}$ is a nondegenerate map $\mathcal{Q}: A^{+} \rightarrow H^{+}$and $D(H) \cong H \bowtie H$, which as an algebra is a tensor product $H \otimes H$, see [19] where the forward direction of the isomorphism was proven for the first time. So the crossed module structures $\mathcal{L} \subset$ $H^{+}$that we must classify become submodules of $A^{+}$under the action of $H$ from the left and the right (viewed as left via $S$ ), which we computed as the left and right coregular ones. Converting back to $\Lambda^{1}$ means of course 2-sided ideals as stated. We note that $1 / 2$ a year after Goslar and several months after [2] was archived as math.QA/9608016, two students of Rosso used this line of proof in a preprint published in [24]. Although written more formally, on critical technical issues such as the twisted variants for $\mathbb{C}_{q}[G]$ the complete non-generic classification is not proven any more than [2]. That was done a year later (by a different method) by Heckenberger and Schmuedgen 25. but for some families of $G$ only, which appears to be the current state of play.

At present we are interested only in the finite-dimensional case where we need only the algebraic theorem as above. Note that every Artinian algebra has a unique block decomposition, which includes all finite-dimensional algebras $H$ over a field $k$. The decomposition is equivalent to finding a set of orthogonal centrally primitive idempotents $e_{i}$ with

$$
1=\sum_{i} e_{i}
$$

These generate ideals $e_{i} H$. Note that $e_{i}^{2}=e_{i}$ implies that $\epsilon\left(e_{i}\right)=0,1$ and the above implies that exactly one is nonzero. Similarly $H^{+}=\oplus_{i} e_{i} H^{+}$is a decomposition of 
$H^{+}$. Hence

$$
\Omega_{u n i v}^{1} \cong \oplus_{i} \Omega_{e_{i}}^{1}
$$

where, for any central projector $e$ we have a calculus

$$
\Omega_{e}^{1}=A \otimes \Lambda_{e}^{1}, \quad \Lambda_{e}^{1}=e H^{+} .
$$

We build the left-invariant 1-forms directly on the block as isomorphic to $\mathrm{H}^{+}$ modulo the kernel of multiplication by $e$. In these terms (tracing through the details of the classification theorem[2]) we have explicitly:

$$
(e h) . a=\sum a_{(1)} e \mathcal{R}_{2}\left(a_{(2)}\right) h \mathcal{R}_{1}\left(a_{(3)}\right), \quad \mathrm{d} a=\sum a_{(1)} e \mathcal{Q}_{1}\left(a_{(2)}\right)-a e ; \quad \theta=e
$$

(the calculus here is inner). We use the Sweedler notation $\Delta a=\sum a_{(1)} \otimes a_{(2)}$ and the notation from [19] where $\mathcal{R}_{1}(a)=(a \otimes \mathrm{id})(\mathcal{R})$ etc. Since $\mathcal{R}_{1}$ is an algebra map and $\mathcal{R}_{2}$ an antialgebra map, one may easily verify that this defines a bimodule and that the Leibniz rule is obeyed. It generalizes the standard Jurco's formulae. Note that in the finite-dimensional semisimple case each block will be the matrix block of an irreducible representation in line with Corollary 4.1. In this case among the projectors there will be exactly one where $e h=e \epsilon(h)$ for all $h \in H$ (the normalized unimodular integral). It has counit 1 and corresponds to the trivial representation; we exclude it in view of $e H^{+}=0$.

This semisimple case is the one that applies in the quantum double example in the next section. In the nonsemisimple case the algebra $H$ has a nontrivial Jacobson radical $J$ defined as the intersection of all its maximal 2-sided ideals. It lies in $H^{+}$. Hence by Theorem 4.2 there is a calculus

$$
\Omega_{s s}^{1}=A \otimes H^{+} / J
$$

which we call the 'semisimple quotient' of the universal calculus. $H / J$ has a decomposition into matrix blocks giving a decomposition of $\Omega_{s s}^{1}$ along the lines of the semisimple case.

Example 4.3. Let $\mathbb{C}_{q}\left[S L_{2}\right]$ be the 27-dimensional reduced quantum group at $q$ a primitive cube root of unity. Here

$$
a^{3}=d^{3}=1, \quad b^{3}=c^{3}=0
$$

in terms of the usual generators. The enveloping Hopf algebra $u_{q}\left(s l_{2}\right)$ is known to have the block decomposition

$$
u_{q}\left(s l_{2}\right)=M_{3}(\mathbb{C}) \oplus B
$$

where $B$ is an 18-dimensional non-matrix block (the algebra is not semisimple) with central projection of counit 1. Hence the universal calculus decomposes into nontrivial calculi of dimensions 9,17 . Moreover, $J \subset B$ is 13-dimensional and the quotient

$$
B / J=\mathbb{C} \oplus M_{2}(\mathbb{C})
$$

implies a 4-dimensional calculus as a quotient of the 17-dimensional one (the other summand $\mathbb{C}$ gives zero). Here $\Omega_{s s}^{1}$ is the direct sum of the 4 and 9 dimensional matrix calculi.

The natural choice of calculus here is 4-dimensional and has the same form as the lowest dimension calculus for generic $q$, the $4 \mathrm{D}$ one first found by hand by Woronowicz [1]. For roots of unity the cohomology and entire geometry are, 
however, completely different from the generic or real $q$ case as shown in [26]. This takes $\mathbb{C}_{q}\left[S L_{2}\right]$ at $3,5,7$-th roots as a finite geometry where all computations can be done and all ideas explored completely. We find, for example, the Hodge ${ }^{*}$ operator for the natural $q$-metric, and show that the moduli space of solutions of Maxwell's equations without sources or 'self-propagating electromagnetic modes' decomposes into a direct sum

$\{$ Maxwell zero modes $\}=\{$ zero - curvature $\} \oplus\{$ self - dual $\} \oplus\{$ anti - selfdual $\}$

of topological (cohomology) modes, self-dual and anti-selfdual ones. The noncommutative de Rham cohomology here in each degree has the same dimension as the space of left-invariant forms, for reasons that are mysterious. We also find that the number of self-dual plus zero curvature modes appears to coincide with the number of of harmonic 1-form modes. We find in general that $\mathbb{C}_{q}\left[S L_{2}\right]$, although finite dimensional and totally algebraic, behaves geometrically like a noncompact manifold. It would be very interesting to understand this in terms of the nonsemisimplicity of $u_{q}\left(s l_{2}\right)$. It would also be interesting to look at the differential geometry induced by taking the other blocks according to our classification theorem, particularly the non-matrix block.

\section{Differentials on the quantum Codouble of A Finite group}

In this section we are going to demonstrate the classification theorem in the previous section for the most famous factorisable coquasitriangular Hopf algebra of all, namely the coordinate algebra of the Drinfeld quantum double itself. So $A=D^{*}(\mathcal{A})=\mathcal{H}^{c o p} \backslash \mathcal{A}$ where $\mathcal{A}$ is a finite dimensional Hopf algebra and $\mathcal{H}$ is its dual. As an algebra the codouble is a tensor product but we are interested in bicovariant calculi on it, which depends on the doubly-twisted coproduct. By our theorem, these are classified by two-sided ideals in $D(\mathcal{H})$. Actually, we compute only the case where $\mathcal{A}=k(G)$, the functions on a finite group $G$, but the methods apply more generally. Then $A=k G>k(G)$. We assume $k$ is of characteristic zero.

Theorem 5.1. Differential calculi on $D^{*}(G)=k G>4 k(G)$ are classified by pairs $(\mathcal{C}, V)$ where $\mathcal{C} \subset G$ is a conjugacy class and $V$ is an irreducible representation of the centralizer, and at least one of $\mathcal{C}, V$ are nontrivial. The calculus has dimension $|\mathcal{C}|^{2} \operatorname{dim}(V)^{2}$.

Proof. Here $H=D(G)=k(G)>\triangleleft k G$ is a semidirect product by the adjoint action. Taking basis $\left\{\delta_{s} \otimes u \mid s, u \in G\right\}$, the product is of course $\left(\delta_{s} \otimes u\right)\left(\delta_{t} \otimes v\right)=$ $\delta_{s, u t u^{-1}}\left(\delta_{s} \otimes u v\right)$. Consider an element of the form $e=\sum_{s} \delta_{s} \otimes e_{s}$ where $e_{s} \in k G$. To be central, we consider

$$
\delta_{t} . e=\delta_{t} \otimes e_{t}, \quad e . \delta_{t}=\sum_{s} \delta_{s} A d_{e_{s}}\left(\delta_{t}\right) \otimes e_{s} .
$$

Writing $e_{s}=\sum_{u} e_{s, u} u$, say, equality requires

$$
e_{t, u} \delta_{t}=e_{u t u^{-1}, u} \delta_{u t u^{-1}}, \quad \forall t, u \in G
$$

This implies that $e_{s} \in k G_{s}$ the group algebra of the centralizer of $s$ in $G$. Next, $u . e=e . u$ requires that $e_{u s u^{-1}}=u e_{s} u^{-1}$ for all $u, s \in G$. Thus central elements $e$ are of the form

$$
e=\sum_{s \in \mathcal{C}} \delta_{s} \otimes e_{s}, \quad e_{s} \in k G_{s}, \quad u e_{s} u^{-1}=e_{u s u^{-1}}, \quad \forall u \in G
$$


for some Ad-stable subset $\mathcal{C} \subset G$. In that case one may compute $e^{2}=\sum_{s} \delta_{s} \otimes e_{s}^{2}$. Hence projectors are precisely of the above form with each $e_{s}$ a central idempotent of $k G_{s}$. For a centrally primitive idempotent we need $\mathcal{C}$ a conjugacy class and a centrally primitive idempotent $e_{0}$ on the group algebra of the centralizer $G_{0}$ (any one point $s_{0} \in \mathcal{C}$ determines the rest). The choice of $e_{0}$ comes from the block decomposition of $k G_{0}$ and in characteristic zero this is given by irreducible representations $V$. The relation is

$$
e_{0}=\frac{\operatorname{dim}(V)}{\left|G_{0}\right|} \sum_{u \in G_{0}} \operatorname{tr}_{V}\left(u^{-1}\right) u
$$

This gives the block decomposition of $D(G)$. For $D(G)^{+}$we remove the trivial case. $\diamond$

Note that this result is exactly in line with Corollary 4.1 since the result is the same data as for the classification of irreducible representations of $D(G)$, which are also the same as irreducible crossed $G$-modules. This is to be expected as $D(G)$ is semisimple because the square of its antipode is the identity. Let $\left\{e_{i}\right\}$ be a basis of $V$ and let $a, b, c$ etc. denote elements of $\mathcal{C}$. Note also that $\mathcal{C}$ is just the data for a calculus $\Omega^{1}(k(G))$ on a finite group function algebra, while $V$ is just the data for a calculus $\Omega^{1}\left(k G_{0}\right)$ on a finite group algebra viewed 'up side down' as a noncommutative space, a result in [2]. The calculus on the double glues together these calculi. We now obtain explicit formulae as follows.

Given the data above, the associated representation $W$ of $D(G)$ has basis $\left\{e_{a i}\right\}$ say, with action

$$
\delta_{s} \cdot e_{a i}=\delta_{s, a} e_{a i}, \quad u . e_{a i}=\sum_{j} e_{u a u-1 j} \zeta_{a}(u)^{j}{ }_{i},
$$

where $\zeta: \mathcal{C} \times G \rightarrow G_{0}$ is a cocycle

$$
\zeta_{a}(u)=g_{u a u^{-1}}^{-1} u g_{a} ; \quad \zeta_{a}(u v)=\zeta_{\text {vav }^{-1}}(u) \zeta_{a}(v)
$$

defined by any section map $g: \mathcal{C} \rightarrow G$ such that $g_{a} s_{0} g_{a}^{-1}=a$ for all $a \in \mathcal{C}$, and we use its matrix in the representation $V$. Completely in terms of matrices, we have the representation of $D(G)$ by

$$
\rho\left(\delta_{s} \otimes u\right)^{a i}{ }_{b j}=\delta_{s, a} \delta_{u^{-1} a u, b} \zeta_{b}(u)^{i}{ }_{j} .
$$

We also need the universal R-matrix and quantum Killing form

$$
\mathcal{R}=\sum_{u \in G} \delta_{u} \otimes 1 \otimes 1 \otimes u, \quad \mathcal{Q}=\sum_{u, v} \delta_{u v u^{-1}} \otimes u \otimes \delta_{u} \otimes v
$$

of $D(G)$. Finally, we need the Hopf algebra structure of $A=k G>k(G)=$ $\left\{s \otimes \delta_{u} \mid s, u \in G\right\}$ with coproduct

$$
\Delta \delta_{u}=\sum_{v w=u} \delta_{v} \otimes \delta_{w}, \quad \Delta s=\sum_{u} s \delta_{u} \otimes u^{-1} s u
$$

and $s, \delta_{u}$ commuting.

We insert these formulae into the Jurco-type construction for a representation $(\rho, W)$ and the conventions of [2]. One can do it either for left-invariant forms $\Lambda$ as elsewhere in the paper, or, which gives nicer results for our present conventions for $D^{*}(G)$, we can do it for right invariant forms, which we denote by $\bar{\Lambda}^{1}$. In this 
case we define $\bar{\Lambda}^{1}=\operatorname{End}(W)$ with basis $\left\{e_{\alpha}{ }^{\beta}\right\}$ and use the standard formulae for quasitriangular Hopf algebras, cf. Section 4:

$$
\begin{gathered}
\Omega^{1}=\operatorname{End}(W) \otimes A, \quad a . e_{\alpha}{ }^{\beta}=\sum \rho\left(\mathcal{R}_{1}\left(a_{(1)}\right)\right)^{\gamma}{ }_{\alpha} e_{\gamma}{ }^{\delta} \rho\left(\mathcal{R}_{2}\left(a_{(2)}\right)\right)^{\beta}{ }_{\delta} a_{(3)} \\
\mathrm{d} a=\sum \rho\left(\mathcal{Q}_{2}\left(a_{(1)}\right)\right) a_{(2)}-\theta a, \quad \forall a \in A . ; \quad \theta=\sum_{\alpha} e^{\alpha}{ }_{\alpha},
\end{gathered}
$$

where $\rho(h)=\rho(h)^{\alpha}{ }_{\beta} e_{\alpha}^{\beta}$ and summations of indices are understood. Putting in the explicit formulae (19)- 211) we have basis $\left\{e_{a i}{ }^{b j}\right\}$ say, and find

$$
\begin{gathered}
\Omega^{1}=\operatorname{End}(W) \cdot D^{*}(G), \quad f e_{a i}{ }^{b j}=e_{a i}{ }^{b j} L_{b}(f), \quad \mathrm{d} f=\sum_{a, i} e_{a i}{ }^{a i} \partial_{a}(f) \\
s e_{a i}{ }^{b j}=\sum_{k} e_{s a s^{-1} k^{b j}} \zeta_{a}(s)^{k}{ }_{i} b^{-1} s b, \quad \mathrm{~d} s=\sum_{a, i, j} e_{s a s^{-1} i}{ }^{a j} \zeta_{a}(s)^{i}{ }_{j} a^{-1} s a-\theta s
\end{gathered}
$$

for all $s \in G, f \in k(G)$. Here $\partial_{a}=L_{a}$ - id, where $L_{a}(f)=f(a())$ denotes lefttranslation in the direction of $a$ and $\theta=\sum_{a i} e_{a i}{ }^{a i}$ makes the calculus inner. The special case where we take the trivial representation of the centralizer is a canonical calculus of dimension $|\mathcal{C}|^{2}$ associated to any conjugacy class, cf. some similar formulae from a different point of view (viewing the double as a bicrossproduct) in 27.

For example, for $S_{3}$ with $u=(12), v=(23), w=(13)$, the possible conjugacy classes are $\{e\},\{u v, u v\},\{u, v, w\}$ of order $1,2,3$. Their centralizers are $S_{3}, \mathbb{Z}_{3}, \mathbb{Z}_{2}$. Hence we have calculi of dimension 1,4 for the first class (the nontrivial irreducibles of $S_{3}$ ), three calculi of dimension 4 (the nontrivial irreducibles of $\mathbb{Z}_{3}$ ) for the second class and two of dimension 9 (the two nontrivial irreducibles of $\mathbb{Z}_{2}$ ) for the third class. Of these, we now focus on the 9-dimensional calculus since the associated conjugacy class of order 3 defines the usual differential calculus on $S_{3}$ and the $D^{*}\left(S_{3}\right)$ calculus is an extension of that. As basepoint we take $s_{0}=u$ and as section we take $g_{u}=e, g_{v}=w, g_{w}=v$. We let $q= \pm 1$ according to the trivial or nontrivial representation of $\mathbb{Z}_{2}$. The resulting cocycle as a function on $S_{3}$ is

\begin{tabular}{c|cccccc}
$S_{3}$ & 1 & $u$ & $v$ & $w$ & $u v$ & $v u$ \\
\hline$\zeta_{u}$ & 1 & $q$ & 1 & 1 & $q$ & $q$ \\
$\zeta_{v}$ & 1 & $q$ & $q$ & 1 & 1 & $q$ \\
$\zeta_{w}$ & 1 & $q$ & 1 & $q$ & $q$ & 1
\end{tabular}

For the calculus, we obtain from the above that $\mathrm{d} f=\sum_{a} e_{a} \partial_{a}(f)$ is the usual calculus on $S_{3}$ with $e_{a} \equiv e_{a}{ }^{a}$, and

$$
\mathrm{d} u=q\left(e_{u} u+e_{w}{ }^{v} w+e_{v}{ }^{w} v\right)-\theta u, \quad \mathrm{~d} v=e_{u}{ }^{w} u+q e_{v} v+e_{w}{ }^{u} w-\theta v
$$

and the same with $v, w$ interchanged in the second expression. Here $\theta=e_{u}+e_{v}+e_{w}$. We also have

$$
\mathrm{d}(u v)=\left(q e_{u}{ }^{w}+q e_{v}{ }^{u}+e_{w}{ }^{v}\right) v u-\theta u v, \quad \mathrm{~d}(v u)=\left(q e_{u}{ }^{v}+e_{v}{ }^{w}+q e_{w}{ }^{u}\right) u v-\theta v u .
$$

Of course, it is enough to work with generators $u, v$ say of $S_{3}$ using the commutation relations from (23), namely

$$
u e_{a}{ }^{b}=q e_{u a u}{ }^{b} b u b, \quad v e_{u}{ }^{b}=e_{w}{ }^{b} b v b, \quad v e_{v}{ }^{b}=q e_{v}{ }^{b} b v b, \quad v e_{w}{ }^{b}=e_{u}{ }^{b} b v b .
$$




\section{Braided APPROACH TO EXTERIOR Algebras AND Hodge *}

Once we have classified and chosen $\Omega^{1}$, we still need the higher exterior algebra $\Omega$. When the coordinate algebra is a Hopf algebra $A$, there is a general construction due to Woronowicz [1] which we discuss in this section. We give a different and more computable construction [6] for it based on braided groups or Hopf algebras in braided categories and in particular the 'braided factorial' matrices introduced in [28]. It was later extended more formally by Bespalov and Drabant in a paper [7] that deserves to be more widely known. In our approach there is a direct geometric meaning related to the Hodge * operator which we would particularly like to explain. We will continue in the right-invariant conventions used in Section 5 but there is an identical theory with the original left-invariant forms.

First of all, the Woronowicz exterior algebra is defined as a quotient of the tensor algebra

$$
\Omega=T_{A}\left(\Omega^{1}\right) / \oplus_{n} \operatorname{ker} A_{n}, \quad A_{n}=\sum_{\sigma \in S_{n}}(-1)^{l(\sigma)} \Psi_{i_{1}} \cdots \Psi_{i_{l(\sigma)}}
$$

where $\Psi_{i} \equiv \Psi_{i, i+1}$ denotes a certain braiding $\Psi: \Omega^{1} \otimes_{A} \Omega^{1} \rightarrow \Omega^{1} \otimes_{A} \Omega^{1}$ acting in the $i, i+1$ place of $\left(\Omega^{1}\right)^{\otimes_{A}^{n}}$ and $\sigma=s_{i_{1}} \cdots s_{i_{l(\sigma)}}$ is a reduced expression in terms of simple reflections. This is well-defined just because $\Psi$ obeys the braid or YangBaxter relations. On the other hand, one can write $\Omega=\bar{\Lambda} \otimes A$ where $\bar{\Lambda}$ is the right-invariant exterior algebra

$$
\bar{\Lambda}=T\left(\bar{\Lambda}^{1}\right) / \oplus_{n} \operatorname{ker} A_{n},
$$

now as a tensor algebra modulo relations over $k$, with $A_{n}$ now defined over $k$ by the crossed module braiding $\Psi: \bar{\Lambda}^{1} \otimes \bar{\Lambda}^{1} \rightarrow \bar{\Lambda}^{1} \otimes \bar{\Lambda}^{1}$. This is the braiding due to the quasitriangular structure of the Drinfeld quantum double in the finite-dimensional case. Note that there is a similar construction based on the dual crossed module $\Lambda^{1 *}$. We similarly define $\Lambda^{*}$ by the tensor algebra on this modulo the kernels of $A_{n}^{*}$. We interpret $\Omega^{*}(A)$ generated by this as the algebra of 'skew tensors fields'.

To explain the geometric meaning consider first the tensor algebras $T\left(\bar{\Lambda}^{1}\right)$ and $T\left(\bar{\Lambda}^{1 *}\right)$ which are dually paired braided groups in a category with braiding $-\Psi$ and with pairing in degree 1 that of $\bar{\Lambda}^{1}$ with $\bar{\Lambda}^{* 1}$. This general type of linear braided Hopf algebras have been introduced by the author and we refer to [19, Ch. 10] for an introduction; in particular, the pairing of a product of $n$ generators of one with $n$ of the other is given by a braided-factorial matrix $[n ;-\Psi]$ ! (these are like $q$-factorials but with $q$ replaced by the braiding $-\Psi$ ) defined by [28] 19:

$$
\begin{gathered}
{[n ;-\Psi] !=(\mathrm{id} \otimes[n-1 ;-\Psi] !)[n ;-\Psi]} \\
{[n ;-\Psi]=\mathrm{id}-\Psi_{12}+\Psi_{12} \Psi_{23}+\cdots+(-1)^{n-1} \Psi_{12} \cdots \Psi_{n-1, n}}
\end{gathered}
$$

We observed in [6] that these $[n,-\Psi]$ ! may be used to define $q$-epsilon tensors on braided groups, and hence the exterior algebra in a 'braided approach' which was taken further and formalized in [7]. Indeed, in the same way as for usual shuffle algebras,

$$
A_{n}=[n ;-\Psi] !
$$

For example,

$$
[3 ;-\Psi] !=(\operatorname{id} \otimes[2 ;-\Psi])[3 ;-\Psi]=\left(\mathrm{id}-\Psi_{23}\right)\left(\mathrm{id}-\Psi_{12}+\Psi_{12} \Psi_{23}\right)=A_{3}
$$

It follows that: 
Proposition 6.1. $\bar{\Lambda}, \bar{\Lambda}^{*}$ are mutually dual braided groups non nondegenerately paired. They are the free tensor algebras $T\left(\bar{\Lambda}^{1}\right)$ and $T\left(\bar{\Lambda}^{1 *}\right)$ as linear braided groups modulo the coradicals of their duality pairing.

We can do the same thing of course with just the $A_{2}=$ id $-\Psi$ relations, which is the quadratic exterior algebra. Then we again have dually paired braided groups $\bar{\Lambda}_{\text {quad }}$ and $\bar{\Lambda}_{\text {quad }}^{*}$ but this time they might not be nondegenerately paired. It can often happen that they are and that the quadratic and Woronowicz quotients coincide, but in theory we need $A_{3}$ etc. to be sure. Also, it is instructive to verify that $\underline{\Delta} x=x \otimes 1+1 \otimes x$ etc., on right-invariant 1-forms indeed extends as a braided group in a category with braiding matrix $-\Psi$ (this is the category of modules of a $\mathbb{Z}_{2}$ extension of the Drinfeld double when $A$ is finite dimensional.) This is a standard computation for linear braided spaces: $\underline{\Delta}\left(\sum_{i} x_{i} y_{i}\right)=\sum_{i}\left(x_{i} \otimes 1+1 \otimes x_{i}\right)\left(y_{i} \otimes 1+1 \otimes y_{i}\right)=$ $\sum_{i}\left(x_{i} y_{i} \otimes 1+1 \otimes x_{i} y_{i}+x_{i} \otimes y_{i}-\Psi\left(x_{i} \otimes y_{i}\right)\right)$ which is zero whenever $\sum_{i} x_{i} y_{i}=0$ in $\bar{\Lambda}_{\text {quad }}$. The only cosmetic difference is that usually one writes the kernel of $A_{2}$ as the image of id $-\Psi^{\prime}$ for some other matrix $\Psi^{\prime}$. Similarly for $\bar{\Lambda}_{\text {quad }}^{*}$ and the pairing. Some of these ideas were later used by Rosso, we refer to [29] for a discussion.

In fact we will see next that, among finite-dimensional exterior algebras of this type, the Woronowicz one should be thought of as the largest one admitting Poincaré duality (in the sense of a Hodge * operator) at the level of forms. As has been emphasized by Connes, the existence of Poincaré duality for classical manifolds is one of their primary characteristics that should be preserved in noncommutative geometry. Thus:

Corollary 6.2. If the exterior algebras $\bar{\Lambda}_{\text {quad }}$ or $\bar{\Lambda}$ are finite-dimensional then they have a unique top form up to normalization. In the case of $\bar{\Lambda}$ it means a nondegenerate Hodge- ${ }^{*}$ map $\bar{\Lambda} \rightarrow \bar{\Lambda}^{*}$.

Proof. Finite-dimensional braided groups $B$, like usual Hopf algebras, admit exactly one translation-invariant integral up to normalization. The proof is exactly the same as for usual Hopf algebras (it has been written out in full in [30]). We apply this theory to $B^{*}=\bar{\Lambda}_{q u a d}$ or $B^{*}=\bar{\Lambda}$. An integral functional on $B$ then means an element 'Top' (say) of $B^{*}$ such that $x$.Top $=\epsilon(x)$ Top for all forms $x$ (where $\epsilon(1)=1$ and $\epsilon=0$ on higher degree is part of the braided group structure), i.e. exactly the property enjoyed by a form of top degree. Hence such a form Top exists and is unique up to scale, under our finite-dimensionality assumption. In this situation there is a general construction for a Hodge-* operator [6], which will be invertible precisely when the duality pairing is nondegenerate.

Note that in the presence of an invariant nondegenerate metric $\eta=\eta^{a b} e_{a} \otimes e_{b}$ (summations understood) identifying $\bar{\Lambda}^{1}$ and $\bar{\Lambda}^{1 *}$, the Hodge * will be a map $\bar{\Lambda} \rightarrow \bar{\Lambda}$ and extends to a bimodule map $\Omega \rightarrow \Omega$. We can also define it perhaps more naively by the usual formulae as follows, but being careful about ordering. Let $d$ be the top degree, then

$$
\begin{gathered}
\epsilon_{a_{1} \ldots a_{d}} \text { Top }=e_{a_{1}} \wedge \cdots \wedge e_{a_{d}} \\
*\left(e_{a_{1}} \wedge \cdots \wedge e_{a_{m}}\right)=d_{m}^{-1} \epsilon_{a_{1} \ldots a_{d}} \eta^{a_{m+1} b_{1}} \cdots \eta^{a_{d} b_{d-m}} e_{b_{d-m}} \wedge \cdots \wedge e_{b_{1}}
\end{gathered}
$$

for some normalisations $d_{m}$, which is the operator used recently in [26] and in other conventions in [31]. Its presence means that we can write down actions such as $-\frac{1}{4} F \wedge * F$ etc. Integration and the Hodge * thus play the role of the operator 
norm \|\| in the operator approach discussed in Section 2. The two approaches were compared in a simple model in 32 .

Finally, we give an example of some of the above. For coquasitriangular Hopf algebras in the conventions of Section 5 we have the standard braiding

$$
\Psi\left(e_{\alpha}{ }^{\beta} \otimes e_{\gamma}^{\delta}\right)=e_{\mu}{ }^{\nu} \otimes e_{\sigma}{ }^{\tau}\left(R^{-1}\right)^{\alpha_{1}}{ }_{\alpha}{ }^{\mu}{ }_{\alpha_{2}} R^{\beta}{ }_{\alpha_{3}}{ }^{\alpha_{2}}{ }_{\gamma} R^{\delta}{ }_{\alpha_{4}}{ }^{\alpha_{3}}{ }_{\tau} \tilde{R}^{\alpha_{4}}{ }_{\nu}{ }^{\sigma}{ }_{\alpha_{1}}
$$

where $R=(\rho \otimes \rho)(\mathcal{R})$ and $\tilde{R}=(\rho \otimes \rho \circ S)(\mathcal{R})$. This is adjoint to the braided-matrix relations of the braided matrices $B(R)$, i.e. $\bar{\Lambda}^{1 *}$ is a standard matrix braided Lie algebra. This is discussed further in [33]. We can compute this braiding explicitly for $D^{*}(G)$ in Section 5 to find:

$$
\Psi\left(e_{a i}{ }^{b j} \otimes e_{c k}{ }^{d l}\right)=e_{a^{-1} b c b^{-1} a m}{ }^{d l} \zeta_{c}\left(a^{-1} b\right)^{m}{ }_{k} \otimes \zeta_{b}^{-1}\left(d^{-1}\right)^{j} p_{d^{-1} a d n}{ }^{d^{-1} b d p} \zeta_{a}\left(d^{-1}\right)^{n}{ }_{i}
$$

where we sum over the matrix indices $m, n, p$ of the representation of the centralizer group. The relations of the exterior algebra are then computed using the braidedfactorial matrices above.

For example, for $D^{*}\left(S_{3}\right)$ with the standard order 3 conjugacy class and either representation $q= \pm 1$ of the isotropy group $\mathbb{Z}_{2}$, we have dimensions and cohomology in low degree:

$$
\begin{aligned}
& \operatorname{dim}\left(\Omega\left(D^{*}\left(S_{3}\right)\right)\right)=1: 9: 48: 198: \cdots \\
& H^{0}\left(D^{*}\left(S_{3}\right)\right)=k .1, \quad H^{1}\left(D^{*}\left(S_{3}\right)\right)=k . \theta
\end{aligned}
$$

which is the same as for an isomorphic bicrossproduct example computed in [27]. We expect the exterior algebra to be finite-dimensional and the Hilbert series to have a symmetric form (our computer did not have enough memory for further degrees). The exterior algebra appears to be quadratic with the relations in degree 2 from (33) as follows. For simplicity we state them only for $q=1$ :

$$
\begin{gathered}
e_{a}^{b} \wedge e_{a b a^{-1}}^{b}=0, \quad\left(e_{a b a^{-1}}{ }^{a}\right)^{2}+\left\{e_{a}{ }^{a}, e_{b}{ }^{a}\right\}=0, \quad \forall a, b \\
e_{u} \wedge e_{v}+e_{v} \wedge e_{w}+e_{w} \wedge e_{u}=0
\end{gathered}
$$

and the conjugate (product-reversal) of this. Here $e_{a} \equiv e_{a}{ }^{a}$ obey $e_{a}^{2}=0$ and we recover precisely the usual $\Omega\left(S_{3}\right)$ as a subalgebra generated by them. In addition, we have

$$
\begin{aligned}
& e_{u}^{v} \wedge e_{v}{ }^{u}+e_{v}{ }^{w} \wedge e_{w}{ }^{v}+e_{w}{ }^{u} \wedge e_{u}{ }^{w}=0 \\
& e_{u}{ }^{v} \wedge e_{u}{ }^{w}+e_{v}{ }^{w} \wedge e_{v}{ }^{u}+e_{w}{ }^{u} \wedge e_{w}{ }^{v}=0
\end{aligned}
$$

and their opposites, and

$$
e_{u} \wedge e_{u}{ }^{w}+e_{u}{ }^{w} \wedge e_{v}+e_{u}{ }^{v} \wedge e_{w}{ }^{u}=0
$$

and all permutations of $u, v, w$ in this equation, plus all their conjugates. 


\section{REFERENCES}

[1] S.L. Woronowicz. Differential calculus on compact matrix pseudogroups (quantum groups). Commun. Math. Phys., 122:125-170, 1989.

[2] S. Majid. Classification of bicovariant differential calculi. J. Geom. Phys., 25:119-140, 1998.

[3] E. Beggs and S. Majid. Quasitriangular and differential structures on bicrossproduct Hopf algebras. J. Algebra, 219:682-727, 1999.

[4] S. Majid and R. Oeckl. Twisting of quantum differentials and the Planck scale Hopf algebra. Commun. Math. Phys. 205:617-655, 1999.

[5] V.G. Drinfeld. Quantum groups. In A. Gleason, ed., Proceedings of the ICM, pages 798-820, Rhode Island, 1987. AMS.

[6] S. Majid. q-Epsilon tensor for quantum and braided spaces. J. Math. Phys., 36:1991-2007, 1995.

[7] Yu. N. Bespalov and B. Drabant. Differential calculus in braided Abelian categories. Preprint, 1997.

[8] A. Connes. Noncommutative Geometry. Academic Press, 1994.

[9] T. Brzeziński and S. Majid. Quantum differentials and the $q$-monopole revisited. Acta Appl. Math., 54:185-232, 1998.

[10] T. Brzeziński and S. Majid. Quantum group gauge theory on quantum spaces. Commun. Math. Phys., 157:591-638, 1993. Erratum 167:235, 1995.

[11] S. Majid. Some remarks on quantum and braided group gauge theory. In W. Pusz, R. Budzynski and S. Zakrzewski, eds., Proceedings of Quantum Groups and Quantum Spaces. Banach Center Publications 40:335-349, 1997.

[12] A. Connes. $C^{*}$ algebres et géométrie différentielle. C.R. Acad. Sc. Paris, 290:599-604, 1980.

[13] A. Connes and M. Rieffel. Yang-Mills theory over quantum tori. Contemp. Math., 62:237, 1987.

[14] P. Hajac and S. Majid. Projective module description of the $q$-monopole. Commun. Math. Phys., 206:246-464, 1999.

[15] T. Masuda, K. Mimachi, Y. Nakagami, M. Noumi, and K. Ueno. Representations of the quantum group $S U_{q}(2)$ and the little q-Jacobi polynomials. J. Funct. Anal., 99:357-387, 1991.

[16] S. Majid and R. Oeckl. Twisting of quantum differentials and the Planck scale Hopf algebra. Commun. Math. Phys., 205:617-655, 1999.

[17] S. Majid. Quantum and braided group Riemannian geometry. J. Geom. Phys., 30:113-146, 1999.

[18] S. Majid. Riemannian geometry of quantum groups and finite groups with nonuniversal differentials. Commun. Math. Phys., 2002.

[19] S. Majid. Foundations of Quantum Group Theory. Cambridge Univeristy Press, 1995.

[20] S. Majid. A Quantum Groups Primer, L.M.S. Lect Notes vol. 292. Cambridge University Press, 2002.

[21] S. Majid. Advances in quantum and braided geometry. In H.-D. Doebner and V.K. Dobrev, eds., Quantum Group Symposium at Group XXI, pp. 11-26. Heron Press, Sofia, 1997.

[22] B. Jurco. Differential calculi on quantized Lie groups. Lett. Math. Phys. 22:177-186, 1991.

[23] K. Schmüdgen and A. Schüler. Classification of bicovariant differential calculi on quantum groups. Commun. Math. Phys., 170:315-335, 1995.

[24] P. Bauman and F. Schmidt. Classification of bicovariant differential calculi over quantum groups (a representation-theoretic approach). Commun. Math. Phys., 194:71-86, 1998.

[25] I. Heckenberger and K. Schmüdgen. Classification of bicovariant differential calculi on the quanutm groups $S L_{q}(n+1)$ and $S p_{q}(2 n)$. J. Reine Angew. Math., 502:141-162, 1998.

[26] X. Gomez and S.Majid. Noncommutative cohomology and electromagnetism on $\mathbb{C}_{q}\left[s l_{2}\right]$ at roots of unity. Lett. Math. Phys., 2002. In press.

[27] F. Ngakeu, S. Majid, and J-P. Ezin. Classification of differentials and Cartan calculus on bicrossproducts. Preprint, 2002.

[28] S. Majid. Free braided differential calculus, braided binomial theorem and the braided exponential map. J. Math. Phys., 34:4843-4856, 1993.

[29] S. Majid. Braided geometry and the inductive construction of Lie algebras and quantum groups. In Deformation Theory, Symplectic Geometry and Applications, pp. 339-344. Kluwer, 1997.

[30] V.V. Lyubashenko. Modular transformations for tensor categories. J. Pure and Applied Algebra, 98:279-327, 1995 . 
[31] S. Majid and E. Raineri. Electromagnetism and gauge theory on the permutation group $S_{3}$. J. Geom. Phys., 2002. In press.

[32] S. Majid and T. Schuker. $z_{2} x z_{2}$ lattice as a Connes-Lott-quantum group model. J. Geom. Phys., 2002. In press.

[33] X. Gomez and S.Majid. Braided Lie algebras and bicovariant differential calculi over coquasitriangular Hopf algebras. Preprint, 2001.

School of Mathematical Sciences, Queen Mary, University of London, 327 Mile End RD, LONDON E1 4NS, UK 